\title{
LAS NACIONES UNIDAS A FINALES DEL SIGLO XX ${ }^{1}$
}

\author{
Javier Sánchez Cano \\ Investigador en Relaciones Internacionales \\ Centre d'Estudis sobre la Pau i el Desarmament \\ Universitat Autònoma de Barcelona
}

Resum

L'article descriu la creacio $i$ el desenvolupament de l'Organització de Nacions Unides, la bistoria de la qual està marcada des dels seus inicis per la situació de bloqueig en que el conflicte Est-Oest posa al Consell de Seguretat. Es posen de relleu els factors que originen el canvi en el caracter de les activitats de l'Organitzacio, i les contradiccions que la porten a la crisi, pressupostària i d'objectius, de l'última decada. S'expliquen les propostes de reforma, amb una especial mencib a les que formula Maurice Bertrand, que apareixen en un context d'interdepedencia creixent que fa més gran la consciencia i la necessitat objectiva de gestió dels problemes globals.

La fi de la guerra freda comporta la possibilitat que el Consell sigui actiu en temes compromesos de pau i seguretat; la seva actuació després de la invasió de Kuwait per Traq aixt ho va posar de manifest. Els primers anys noranta ban estat marcats, tant per lincrement en el treball sobre aquestes quiestions (augment del nombre de mussions de manteniment de la pau), com per les noves activitats que es demanen de l'Organitzacib, sense precedents en epoques anteriors, $i$ que es deuen, en gran mesura, a lestat generatizzat de transició del sistema internacional.

L'article finalitza amb una reflexió sobre els temes pendents del funcionament de NN.UU. i les perspectives immediates de l'organitzacio.

Resumen

El articulo describe la creación y el desarrollo de la Organizacion de Naciones Unidas, marcado desde sus intcios por la situación de bloqueo en que el conflicto Este-Oeste sume al Consejo de Seguridad. Se señalan los factores que llevan al cambio en el carácter de las actividades de la Organización, y las contradicciones que la llevan a la crisis, presupuestaria y de objetivos, de la pasada década. Se da cuenta de las propuestas de reforma, haciendo especial mencion a las que formula Maurice Bertrand que aparecen en un contexto de interdepedencia creciente que bace mayor la conciencia y la necesidad objetiva de gestion de los problemas globales.

El fin de la guerra fría trae consigo la posibilidad de que el Consejo sea activo en temas comprometidos de paz y seguridad; su actuación tras la invasion

1. Este artículo está parcialmente basado en la Memoria de licencjatura del autor, dirigida por Rafael Grasa y presentada en la Facultad de Cienctas Polficicas y de Sociología de la UAB en septiembre de 1992. 
"Papers": Revista de Sociologia

de Kuwait por Yraq así lo puso de manifiesto. Los primeros años noventa han estado marcados, tanto por el incremento en el trabajo sobre estas cuestiones (aumento del número de misiones de mantenimiento de la paz), como por las nuevas actividades que se demandan de la Organización, sin precedentes en épocas anteriores, $y$ que se deben en gran modo al estado generalizado de transición del sistema internacional.

El articulo finaliza con una reflexión sobre los temas pendientes del funcionamiento de NN.UU. y las perspectivas inmediatas de la Organizacion.

Abstract

The article describes the origin and development of the United Nations Organization, whose main initial feature is the stalemate of its Security Council as a direct consequence of the cold war. The author points out the factors that led to the basic change in the Organization's range of activities, and the contradictions that brought about the crisis of the last decade, both in terms of $f$ nance and goals. The different proposals for institutional reform are mentioned, with a special emphasis on those put forward by Maurice Bertrand. These appear in a growingly interdependent environment, thus increasing both the perception and objective need for global management.

The end of the cold war means greater possibilities for actions undertaken by the UN Security Council, in the freld of peacekeeping and security, as shown by its attitude during the recent Iraqi-Kuwaiti crisss. In the early 90 s the work on these questions has steadily risen (higher number of peacekeeping operations). Furthermore, UN receives new and pressing requirements from different member states, prompting the Organization into unprecedent actions. These needs owe to the general state of transition in the present international system.

Finally, the article tackles a set of unsolved issues related to the UN's functioning and immediate prospects for the work of the Organization.

El fin de la guerra fría ha eliminado el gran lastre que hizo que, durante los últimos cuarenta años, Naciones Unidas (NN.UU.) no pudiera desempeñar el papel que para la Organización concibieron los firmantes de su Carta. Después de décadas de niveles mínimos de actividad, hoy cl Consejo de Seguridad es capaz de aprobar resoluciones sobre los temas más impensables en el pasado. La Organización recibe crecientes demandas de asistencia por parte de gobiernos, por lo que debe no sólo aumentar el número de misiones de mantenimiento de la paz operativas (véase anexo 1), sino organizar otras actividades, antes escasas o inexistentes: preparar y supervisar elecciones, verificar y controlar la destrucción de armamentos y posibles planes nucleares, o proveer asistencia humanitaria.

Este tipo de actuaciones, relativas a cuestiones de la guerra y la paz, nos ha hecho olvidar que la Organización tiene otras importantes carpetas abiertas. Temas que, durante la época de bloqueo del Consejo de Seguridad, constituían el grueso de su trabajo: desarrollo, medio ambiente, demografia, 
etc. Es decir, los llamados problernas globales, cuya importancia - y necesidades de gestión - no dejan de ir en aumento. Hace unos años se planteaba la reforma de la Organización para, precisamente, mejorar su funcionamiento en temas sociales y económicos. Hoy, el cambio dramático en el sistema internacional ha hecho que se le presenten otros problemas urgentes y que se amplíe su ámbito de actuación, lo que varía las perspectivas de revisión del sistema. Algo parece cierto: las Naciones Unidas se han consolidado, y las posibilidades que se abren antes ellas no sólo son amplias, sino también prometedoras. Pero para tener una mejor idea de su futuro es necesario revisar su historia y los cambios que han tenido lugar en el contexto internacional.

\section{PRIMEROS DESARROLLOS DE LA ORGANIZACIONN}

Naciones Unidas nace en unas coordenadas históricas muy precisas: los líderes de los estados aliados en la II Guerra Mundial se plantean, ya antes del fin del conflicto, la creación de una organización internacional de segunda generación, que supere las deficiencias de la anterior Sociedad de Naciones (que funcionó en el período de entreguerras, y no fue capaz de evitar la escalada bélica que desembocó en la Segunda Guerra Mundial). El mantenimiento de la paz y la seguridad internacionales iban, pues, a ser los objetivos centrales de la Carta fundacional de Naciones Unidas, que es frmada en San Francisco el 26 de junio de 1945 por cincuenta y un estados, la práctica totalidad de los del mundo en aquellos días. Quedan excluidos aquéllos vinculados a las potencias del Eje, así como éstas mismas.

La Otganización nace con el acuerdo unánime - aunque parcial- respecto a tres principios básicos:

- El establecimiento de un sistema que funcione a dos niveles separados: la Organización de Naciones Unidas (ONU) debía ocuparse de la cooperación política, mientras que los temas de cooperación en materia económica y social se tratarían desde los organismos especializados. Juntos, ambos niveles forman el llamado Sistema de Naciones Unidas (véase cuadro 1 en pág. 88).

- La instauración de un cierto equilibrio entre la concesión de liberrades a los territorios no autónomos y los intereses estratégicos de las potencias coloniales.

- El papel preeminente que se concede a las grandes potencias en los temas de paz y seguridad internacionales, preeminencia que se deriva de la "responsabiliad especial" que se les otorga en la cuestión. 
"Papers»: Revista de Sociologia

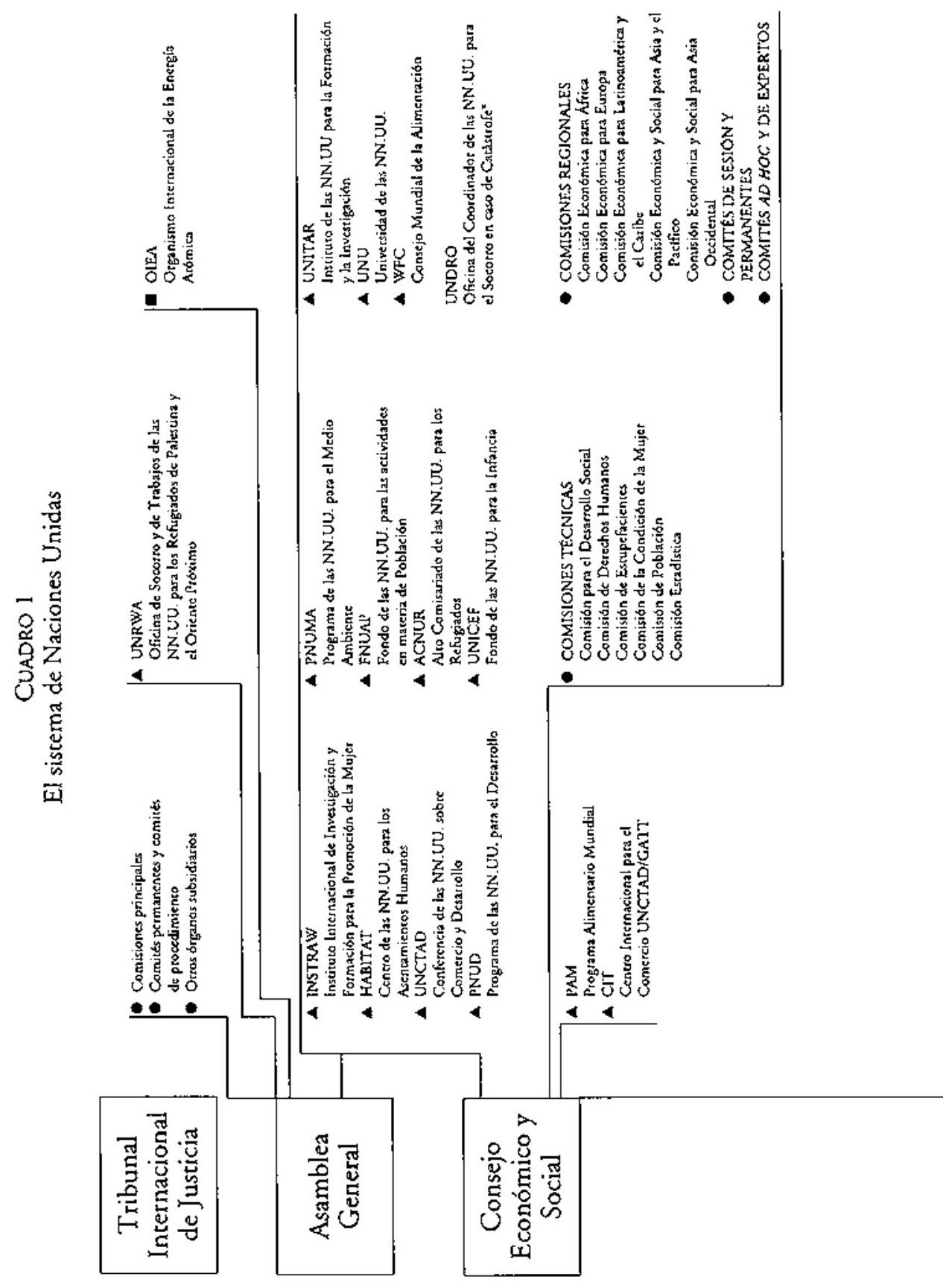


Las Naciones Unidas a finales del siglo xx

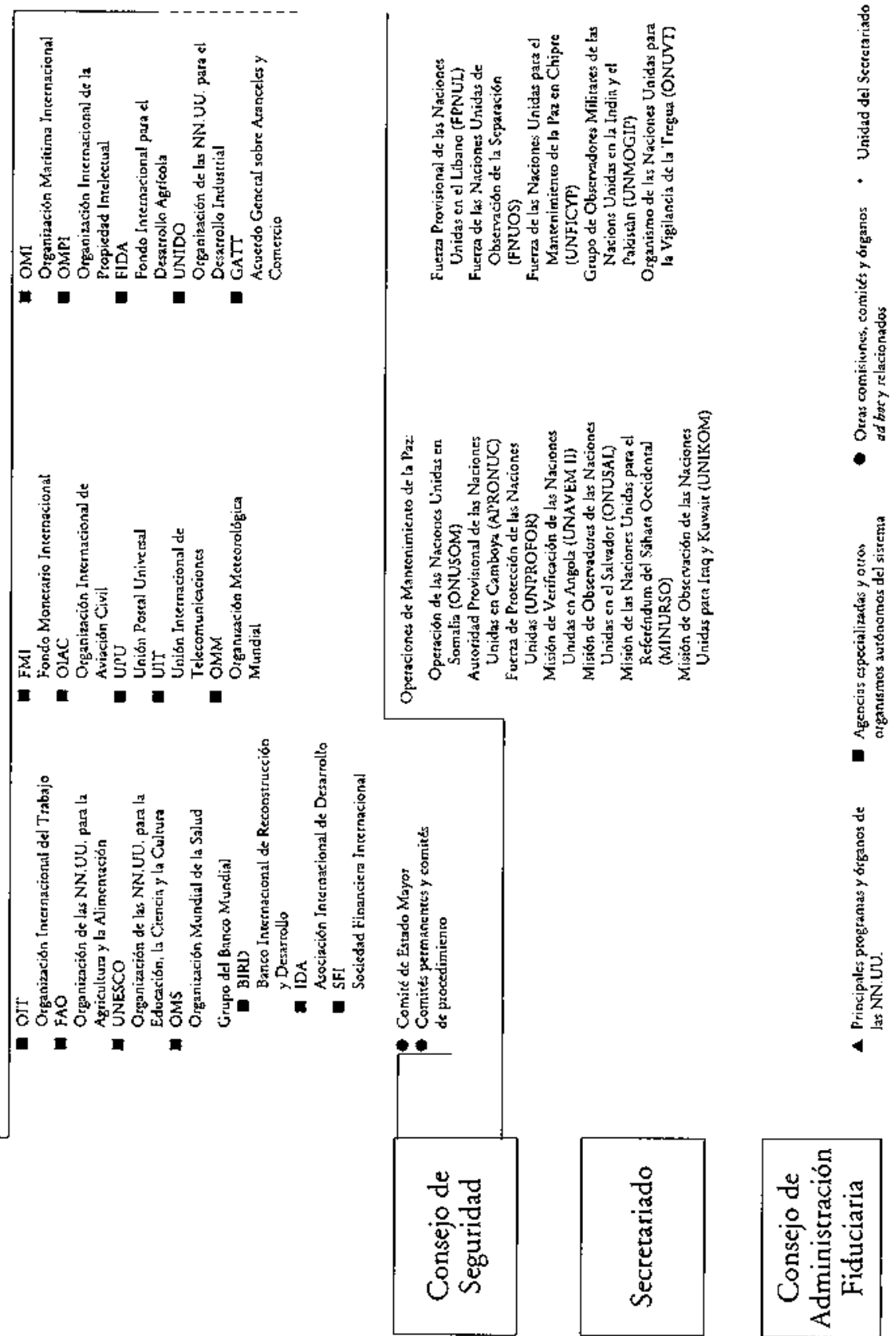


Es así que NN.UU. combina, por una parte, la universalidad que significa la representación de todos los estados, que cuentan con un voto en el órgano plenario --la Asamblea General- donde las decisiones se toman por mayoría (de dos tercios para cuestiones presupuestarias, y simple para otros temas); con la efectividad y el realismo del órgano restringido - el Consejo de Seguridad-, del que forman parte las cinco grandes potencias vencedoras en la II Guerra Mundial (EUA, URSS, Francia, Reino Unido y China), y que quiere reflejar el reparto real del poder mundial. El Consejo, que funciona de modo permanente y está encargado de los temas relativos a la seguridad internacional, toma sus decisiones (cuando se trata de cuestiones sustanciales) con el voto afirmativo de nueve de sus quince miembros (incluido el de las cinco potencias o miembros permanentes).

El modelo perseguido era el de la seguridad colectiva según el cual la seguridad no es un bien que sólo alcanza un estado a expensas de otro, sino que es conseguida mediante un sistema de cooperación política en el que todos comparten unos principios y unas reglas de comportamiento. Los estados actuarían automática y colectivamente contra los transgresores de estas reglas, con medios militares si fuera necesario. La guerra se convierte así en un bien común, y no en un instrumento de política nacional (Groom, 1988:17).

Sin embargo, la guerra fría pervierte la lógica del sistema. Pese a que la ONU (su Primera Comisión encargada de asuntos políticos y de seguridad) tenía como tareas el control de la energía atómica y la prevención de la proliferación nuclear, una tras otra las cinco grandes potencias se dotan de armas nucleares. Asimismo el art. 52 de la Carta, que autoriza la creación de organizaciones regionales de carácter defensivo, abre la puerta, en lógica de guerra fría, a la creación de las organizaciones del Tratado del Atlántico Norte (OTAN, 1949) y del Tratado de Varsovia (OTV, 1955).

El cleavage Este-Oeste marca una época de bajos niveles de cooperación. Dado que Naciones Unidas configura un sistema basado en el acuerdo y la cooperación entre países, el conflicto Este-Oeste tuvo efectos extremadamente paralizantes, sobre todo en aquellos foros en los que era necesario el actuerdo político sobre temas sensibles; así, por ejemplo, se hace imposible emprender acciones de imposición o mantenimiento de la paz desde el Consejo de Seguridad.

Limitado el papel del Consejo en temas de seguridad, va a ser el Secretario General Dag Hammarksjöld (1953-1961) y la Asamblea quienes tomen la iniciariva: se crean así las misiones de mantenimiento de la paz: medidas no coercirivas y sí preventivas, basadas en el consentimiento o voluntariedad, tanto de los estados que aportan tropas como de los que las reciben en su territorio. Las misiones de paz no buscan la imposición de soluciones militares, sino atemperar las tensiones y facilitar salidas negociadas y protección a los civiles. 
Esta actividad, la aportación más notoria de NN.UU. a la paz y la seguridad, genera gastos importantes cuya financiación ha sido objeto de controversia. Assi la ex-Unión Soviética y Francia se negaban a contribuir, aduciendo que no reconocían la competencia de la Asamblea en la materia ni, por ello, su potestad para adoptar decisiones presupuestarias obligatorias ${ }^{2}$.

\section{DEL NUEVO PAPEL DE NN.UU. A LA CRISIS PRESUPUESTARIA}

Es, no obstante, el proceso de descolonización el que ha tenido consecuencias más profundas para el funcionamiento de la Organización. NN.UU. incorpora a los nuevos estados a la comunidad internacional, dotándolos tanto de legitimación como de la posibilidad de hacerse oír al más alto nivel. De los cincuenta y un estados originarios, los miembros de la Organización pasarán a ciento cuarenta y cuatro en 1975 (veáse cuadro 2 en pág. 92). Esta nueva mayoría en la Asamblea General (que se integra, en su mayor parte, en el movimiento de países no alineados) impulsa decididamente el tratamiento de los temas que considera prioritarios: desarrollo, educación, salud, régimen de intercambios desigual. La falta de acuerdo, tanto en la ONU como en otros foros, sobre el tratamiento a dar a estas cuestiones inicia lo que va a convertirse en el conflicto Norte-Sur, que desde los años setenta se añade, y en ocasiones desplaza, al conflicto Este-Oeste.

En general, los nuevos estados quieren que Naciones Unidas funcione tanto como foro desde el que expresar sus posturas, negociar y formular programas, como actor implementador y ejecutor de esas decisiones sobre el terreno: la Organización sería un agente para el cambio y el progreso económico y social. Su conducta en los diferentes órganos va siempre encaminada a este fin; así impulsan el examen de los temas citados; favorecen la creación de nuevos órganos y agencias, y el aumento de miembros en los existentes ${ }^{3}$. Intentan que órganos como las instituciones de Bretton Woods (Fondo Monetario Internacional, FMI y Banco Mundial, BM) y el Consejo de Seguridad se comprometan en temas de desarrollo y se pronuncien sobre prácticas de terceros países (como Israei o Sudáfrica). El presupuesto de la Organización crece, y la parte más importante de éste (alrededor del 70\%) es destinada a proyectos de desarrollo. No solamente el sistema se hipertrofia; también cambia, de hecho,

2. Esta discusión dio lugar a una senzencia del Tribunal Internacional de Justicia, de 20 de julio de 1962, según la cual la Asamblea riene, en virtud del art. 14 de la Carta, derecho a emprender estas misiones y a fijar la forma en que los estados deberán financiatlas.

3. El númeto de miembros no permanentes del Consejo de Seguridad pasa, en 1963, de once a quince. Igualmente, el Consejo Económico y Social pasa de dieciocho a veintisiete miembros, y alcanza en 1971 la actual cifra de cincuenta y cuatro. 
"Papers»: Revista de Sociologia

\section{CUADRO 2}

Evolución del número de Estados miembros, por continentes.

Los porcentajes se refieren a la participación en el número total de miembros

\begin{tabular}{lllrrrr} 
& Europa & América & \multicolumn{1}{c}{ Asia } & \multicolumn{1}{c}{ África } & Oceanía & NN.UU. \\
1945 & $14(27 \%)$ & $22(43 \%)$ & $9(18 \%)$ & $4(8 \%)$ & $2(4 \%)$ & 51 \\
1955 & $26(34 \%)$ & $22(29 \%)$ & $21(28 \%)$ & $5(7 \%)$ & $2(3 \%)$ & 76 \\
1965 & $28(24 \%)$ & $24(20 \%)$ & $27(23 \%)$ & $37(32 \%)$ & $2(2 \%)$ & 118 \\
1975 & $30(21 \%)$ & $30(21 \%)$ & $34(24 \%)$ & $46(32 \%)$ & $4(3 \%)$ & 144 \\
1985 & $30(19 \%)$ & $36(23 \%)$ & $36(23 \%)$ & $50(32 \%)$ & $7(4 \%)$ & 159 \\
1993 & $36(20 \%)$ & $37(21 \%)$ & $48(27 \%)$ & $51(28 \%)$ & $7(4 \%)$ & 179
\end{tabular}

Distribución gráfica por continentes, 1945-1993. Los Estados de Oriente Medio se incluyen en Asia. Se toman los Urales como frontera oriental europea, y se incluye a la URSS en este continente.

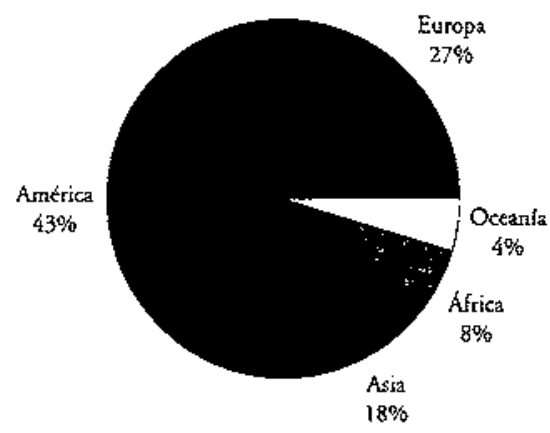

1945

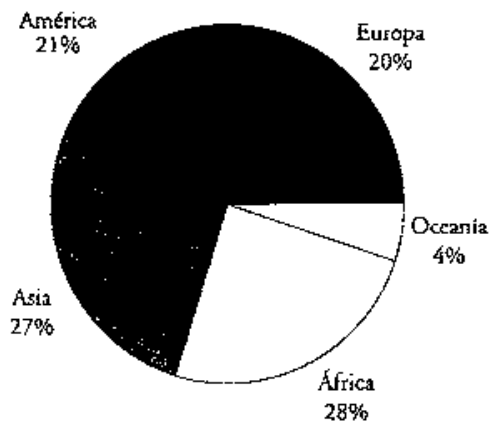

1993

el carácter con que fueron concebidas las agencias de NN.UU.: un ejemplo es la UNESCO, que de su concepción inicial como red de circulación de élites culturales se convierte en un centro impulsor de campañas de alfabetización en los paises menos avanzados.

La reacción de los países del Norte (orientales y occidentales) es similar: aunque los primeros coinciden a menudo, declaratoriamente, con las posturas tercermundistas, tienden en realidad y junto a Occidente a adoprar una 6 ptica restrictiva del papel de NN.UU. Los niveles de concertación en el interior del sistema son bajos; las instituciones de Bretton Woods, controladas por los paises ricos, se disocian de toda iniciativa de desarrollo conjunta con NN.UU. El multilateralismo entra en crisis (Pérez de Cuéllar, 1982:4-5), y las decisiones importantes se toman fuera de las organizaciones del sistema (Renninger, 1989:4). A medio plazo, decrece la popularidad y la confianza en NN.UU., tanto entre los gobiernos occidentales como entre sus poblaciones. Fruto de 


\section{CUADRO 3}

Principales contribuyentes al presupuesto de la Organización

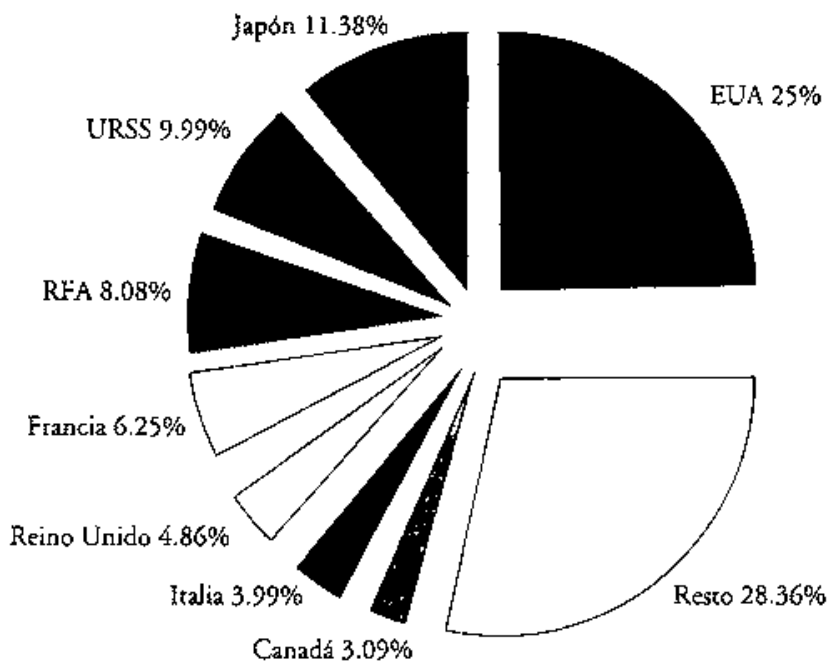

La escala de cuotas se refiere a la participación del Estado en el total del presupuesto ordinario de la Organización. Los datos corresponden a 1990; la cuota de la RDA era del 1,28\%. Bielorrusia y Ucrania hacian ya sus propias contribuciones: 0,33 y $1,25 \%$. Entre el 1 y el $2 \%$ están Arabia Saudita, Australia, Bélgica, Brasil, España, Paises Bajos y Suecia. Las cuotas del resto son inferiores al $1 \%$.

este descontento es el anuncio que realizan en 1983 los EUA de que se retirarán de la UNESCO desde el 1 de enero de 1985; y es, sobre todo, la decisión del Congreso de ese país de reducir su aportación al presupuesto de la Organización lo que precipita la crisis: el Congreso de los Estados Unidos, con argumentos de mala gestión y politización en el funcionamiento de Naciones Unidas, decide en enero de 1985 (enmienda Kassebaum) reducir en un 20\% su aportación al presupuesto de la Organización, a menos que se adopte en. la Asamblea un mecanismo de voto ponderado a las contribuciones económicas. A esta decision siguieron otras el mismo año (enmienda Sundquist, Acta Gramm-Rudman) que redujeron la aportación no en un 20 sino en un $50 \%$ Ésta pasa a ser en 1986 de 100 millones de dólares, dejando impagados orros 110 millones. En realidad, las protestas de Estados Unidos no se deben a lo excesivo del volumen de sus pagos (ciertamente insignificante dentro del presuesto federal), sino a un desacuerdo básico respecto al papel que debe desempeñar la Organización. Como es de esperar, el recorte de más de un $50 \%$ en las aportaciones del estado con mayor cuota ( $25 \%$ del total, véase cuadro 3 ), 
combinado con la rigidez en los sistema de financiación de Naciones Unidas, sumen a la ONU en una crisis financiera profunda.

Para intentar salvar esta situación, Naciones Unidas convocó apresuradamente, en diciembre de 1985, el llamado Grupo de los dieciocho. Sus miembros eran expertos, nombrados de forma representativa, que debian llegar a un compromiso sobre medidas de reducción de gastos y forma de decisión del presupuesto. El grupo redactó un informe (Bertrand 1989a:107-116) en el que se respetaban especialmente las demandas del delegado estadounidense (informe que asume la Asamblea en su resolución 41/213). Sin embargo y pese al mencionado respeto de las demandas de Washington, el Congreso de los EUA no permitió a su gobierno la vuelta a anteriores niveles de contribución. Aunque se había acordado, entre otras cosas, la reducción del número de altos cargos y la aprobación del presupuesto por consenso, los motivos de la queja estadounidense ( $y$ de la mayoría de Estados occidentales, aunque no lo manifestaran abiertamente) no podían satisfacerse con medidas parciales, sino con la revisión de las funciones de NN.UU.

Como afirma Renninger (1989:238), esta conducta de los EUA puede estar justificada, y de hecho actúa como catalizador de la reforma; pero tras ella no existen ni voluntad real de liderazgo ni la propuesta de un modelo bien definido. Es por ello que no aporta ningún elemento nuevo a las teorías de la reforma que, conscientes de este desacuerdo básico Norte-Sur, habían iniciado una seria tarea de análisis y formulación de propuestas desde principios de la década.

\section{PROPUESTAS DE REFORMA}

Es Maurice Bertrand, antiguo miembro de la Unidad de Inspección Conjunta de las NN.UU., quien ha hecho más y mejores aportaciones a la cuestión de la reforma de la Organización, especialmente en sus colaboraciones con el Instituto de las NN.UU. para la Formación y la Investigación (UNITAR). Sus propuestas parten de un gran conocimiento del funcionamiento interno de NN.UU., y son a la vez realistas e innovadoras. Ello, y que su descripción sirve para ilustrar de manera general los problemas existentes en el interior de la Organización, justifica el espacio que se les va a dedicar en este artículo.

Según Bertrand (1989a:52-53), hay tres grandes falacias que impiden el funcionamiento del sistema: las creencias de que i) el camino hacia la paz puede ser el directo "mantenimienro" de ésta por un Consejo que incluya a representantes de las potencias principales; ii) se pueden llevar adelante negociaciones para modificar o mejorar el consenso mundial sin primero definir 
estructuras negociadoras adecuadas a los problemas a resolver y aceptadas por todos los participantes; y iii) es posible favorecer el desarrollo de los más necesitados destinando a ello recursos escasos y distribuidos en forma sectorial y escasamente integrada.

La frase reiterada "falta de voluntad política", utilizada para dar cuenta de todos los males de NN.UU., esconde el hecho de que nadie se ha tomado la molestia de definir qué es y qué no es posible tratar desde la Organización. Se han apoyado retórica y ritualmente las viejas ideas falaces, a través de un discurso siempre cargado y grandilocuente. Desde 1945, el arsenal de éstas incluso se expande: la razonabilidad del uso de la red de organismos especializados en la promoción del desarrollo, y la idea de que el aumento de miembros de NN.UU. atumenta su universalidad sin ir en detrimento de su capacidad negociadora y decisora. No se han explotado sistemáticamente las áreas en que existe convergencia de opiniones y podrían haber sido temas de avance; por el contrario, se trabaja en múltiples frentes en los que no existe acuerdo, por lo que los esfuerzos desplegados no producen resultados significativos. El tiempo y la regularidad hacen que NN.UU. se constituya, no obstante lo inapropiado de estos mecanismos, como un foro político que puede servir de punto de partida a un sistema de paz. Para Bertrand la Organización internacional posee unas características que la hacen imprescindible; pero es necesaria la reforma: Naciones Unidas debe convertirse en una Organización de tercera generación, y se debe iniciar una campaña de medidas creadoras de confianza que modifiquen las relaciones entre gobiernos y las de éstos con sus pueblos.

Esta primera visión de Bertrand está marcada por el convencimiento de la imposibilidad de acuerdo en el Consejo de Seguridad, por lo que los temas económicos deben ser prioritarios para NN.UU.; a partir del avance en éstos, y al modo neofuncionalista, el trabajo para la paz mejorará. Propone no modificar la composición del Consejo de Seguridad, y reestructurar completamente los órganos dedicados a temas económicos y de desarrollo, así como las relaciones de éstos con las instituciones de Bretton Woods. Su idea es conseguir no un foro donde todo el mundo tenga un voto, sino uno donde un número limitado de partes, que representen al total de regiones e intereses del globo, se reúna a alto nivel (ministros de economía) y pueda decidir. Este órgano debería ser el nuevo Consejo Económico y Social (ECOSOC), y para él ofrece Bertrand un ejemplo fórmula de representación: contaría con los doce países de mayor peso mundial (los diez de PNB mayor que el $2.5 \%$ del PNB mundial, más los dos de más de 100 millones de habitantes no incluidos en el grupo anterior). A ellos se ańadirían otros once representantes por regiones mundiales. El Consejo contaría con un secretariado internacional, altamente cualificado, y ambos trabajarían de modo similar al sistema de la CE Consejo (decisores estatales)-Comisión (implementadores comunitarios, defensores del interés de la Organización). Lo más 
importante es que Bertrand propone que su trabajo sea en base territorial, regional y subregional, aunando en cada lugar los diversos sectores -organismos especializados- actuales, y acabando así con su falta de coordinación.

Los teóricos de la reforma matizan sus teorías a medida que evoluciona el contexto internacional. Es claro que el hecho que más influye en el funcionamiento de la Organización va a ser el cambio en la postura soviética respecto a ella, que queda reflejado de forma estructurada en el artículo que Gorbachov publicó en Pravda en septiembre de 1987. En él, el entonces presidente de la Unión Soviética señala que la creciente interdependencia mundial requiere un foro donde gestionar los problemas globales, y que NN.UU. debe convertirse en un centro operativo de ese tipo. Su escrito se confirma en la práctica con el inicio del pago de los atrasos en las contribuciones soviéticas al presupuesto de la Organización; Gorbachov habla asimismo de que la URSS pueda participar en las organizaciones de Bretton Woods, para lo que impulsaría políticas que permitiesen la convertibilidad del rublo en el mercado internacional.

Esta evolución en las posturas soviéticas tiene reflejo en las nuevas propuestas de Bertrand. En un segundo texto (1989b), en el que hace un análisis de las diferentes concepciones entre el Norte y el Sur en su concepción de la seguridad, y señala la utilidad que tiene el realismo dominante en el acercamiento al tema de la reforma por parte de las potencias principales, que muestra que todo cambio deberá ser impulsado por ellas. Bertrand plantea tres ámbitos de necesaria refexión:

1. cómo conseguir capacidad negociadora al nivel de representación adecuado, tema que puede afectar a los grandes tabuies de NN.UU.: la reforma de la Carta y la soberanía e igualdad estatal;

2. la estructura regional: la necesidad de paliar la balcanización actual del sistema mundial de estados a través de formas de cooperación regional al estilo de Europa occidental;

3. cómo superar la enorme sectorialización del sistema, con múltiples agencias especializadas sin enlaces, coordinación ni liderazgo efecrivo entre ellas; y cómo asociar los trabajos de las agencias de la ONU y de Bretton Woods.

En general, se puede afirmar que el conjunto de teóticos de la reforma identifica una serie de creencias erróneas que han lastrado el correcto funcionamiento de la Organización:

1. el que la II Guerra Mundial había creado una especie de "comunidad mundial" que impediría futuros conflictos, que serían prevenidos por un sistema de seguridad colectiva; 
2. que las estructuras parlamentarias nacionales son trasladables al entorno supranacional;

3. la centralidad de los estados-nación como actores en el sistema internacional;

4. La posibilidad del crecimiento y el progreso prácticamente ilimitados.

Comparten igualmente el convencimiento de la necesidad de delimitar espacios de convergencia, para concentrar el trabajo en éstos: por una parte, reconocer las limitaciones del funcionamiento del sistema de seguridad; por otra, abundar en la esfera económica. Es clara la necesidad tanto de que la Organización ofrezca programas de interés para el conjunto de estados, no sólo para los más pobres, como de contar con el apoyo de las potencias principales para plantear cualquier reforma. Una propuesta especialmente destacable es la modificación de los esquemas de cooperación entre estados, que pasa por acabar con la voluntariedad en las aportaciones económicas y eliminar el concepto de ayuda al desarrollo. Los organismos deben trabajar en base a presupuestos acordados, de forma que desaparezca todo rastro de mendicidad o caridad, y desarrollar programas en la totalidad de los estados, de forma que todos tengan intereses objetivos en el buen funcionamiento del sistema.

Todas las propuestas de cambio tienen una variable prácticamente fija: la dificultad de las tareas de mantenimiento de la seguridad a causa del bloqueo del Consejo de Segtridad.

\section{GRANDES CAMBIOS EN EL SISTEMA INTERNACIONAL. EL DESBLOQUEO DEL CONSEJO}

De forma inesperada y en un corto plazo, la nueva postura soviética permite el avance o la resolución de temas abiertos hacía tiempo: el fin del conflicto Irán-Iraq, el acuerdo para la independencia de Namibia, o la retirada soviética de Afganistán son éxitos de la diplomacia desarrollada por la Organización y, en concreto, por el Secretario General, Pérez de Cuéllar. Pero la actividad de NN.UU. Ylega a un punto de inflexión en 1990: el 2 de agosto de ese año tiene lugar la invasión de Kuwait por Iraq. El Consejo reacciona de forma inmediata, y aprueba ese mismo día su resolución 660 . Sucesivamente, este órgano aprobará un total de doce resoluciones, la mayoría de las cuales con arreglo al Capítulo VII de la Carta - relativo a medidas que impliquen el uso de la fuerza, y al cual tan raramente se había apelado anteriormente. Sus contenidos van de la condena inicial a la imposición de sanciones, y a la autorización final al uso de la fuerza (resolución 678) y la previsión de 
reparaciones y controles. El desbloqueo del sistema de seguridad se muestra a to largo de todo este conflicto: la resolución 678 se aprueba con los votos en contra de Cuba y Yemen. China se abstiene; prefiere cooperar con los EUA, que lievan la iniciativa, y mejorar su situación comercial tras las sanciones que se le impusieron por la represión de su revuelta democrática interna. A las puertas de su desintegración como estado unitario, los motivos económicos priman tambiér sobre la URSS, que va a concentrarse cada vez más en su política interna y a abandonar acciones exteriores.

El fin de la guerra fría trae consecuencias directas para la Organización: la afluencia de nuevos miembros (véase en el cuadro 2 la evolución de estados del continente europeo y asiático) que surgen de la desintegración de la URSS; el cambio de posturas y sensibilidades en los países de la Europa Central y Oriental, y, más importante, la posibilidad de un diátogo en nuevos términos con Rusia (que sucede en derechos y obligaciones a la URSS). Esto último abre buenas perspectivas de cara al futuro funcionamiento del Consejo de Seguridad. El conflicro Este-Oeste toca, por el momento, a su fin; los antiguos miembros del bloque soviético desmantelan sus estructuras de cooperación económica (Consejo de Asistencia Económica Mutua, CAME) y militar (OTV), acercándose rápidamente a las existentes en Europa occidental: búsqueda de inversiones, de cooperación con la CEE, y diálogo con los miembros de la OTAN a través del nuevo NACC (Consejo de Cooperación del Atlántico Norte). Se puede aftrmar que se refuerza y se amplía el grupo del Norte.

El desbloqueo del Consejo de Seguridad permite vislumbrar posibilidades de mejora en la gestión del problema central —el mantenimiento de la paz-que dio lugar a la creación de la Organización en 1945. Sin embargo, medio siglo más tarde la agenda internacional está marcada por cuestiones cuya gravedad no es inferior a la de los conflictos armados.

Por una parte, se revisan los conceptos de seguridad, de manera que éstos abarquen factores no necesariamente militares (demográficos, económicos y medioambientales, entre otros) susceptibles de convertirse en amenazas para la paz y la seguridad internacionales. Por otra parte, aumenta la interdependencia global y la necesidad de gestión de diferentes problemas comunes. Esta interdependencia - la mundialización de las relaciones internacionales y el lugar que en ellas ocupan los intercambios comerciales-cuestiona elementos, como la primacía de la alta política (temas militares y de seguridad) frente a la baja (temas económicos) y el papel del estado como actor principal en la sociedad internacional. Es así que, mientras que en términos de poder militar es posible hablar de una única potencia mundial que configura un sistema unipolar, en términos de poder económico hay que referirse a la existencia de diversos centros o a una situación de multipolaridad. 
Los cambios en el sistema internacional y el fin del letargo del Consejo de Seguridad llegaron precisamente cuando la conciencia de la interdependencia global se empezaban a aliar a las iniciativas de acción en el dominio económico. La mejora del clima Este-Oeste va a traer primero progresos bilaterales (Tratado INF, 1987) que se extienden rápidamente al ámbito multilateral universal (los comentados éxitos de NN.UU. desde 1988) y europeo (Carta de París, 1990). Es destacable la ausencia de avances económicos globales parangonables a estos ejemplos de cooperación política.

Es por ello que no es de extrañar que tanto Pérez de Cuéllar en su último año de mandato como el nuevo Secretario General --Butros Ghali, que ocupa el puesto desde enero de 1992 - se hayan felicitado de los cambios que han permitido el funcionamiento del Consejo, y hayan propiciado la dedicación de la Organización a labores relacionadas con él. La idea subyacente es que el progreso en este frente deberá ser catalizador de la mejora del funcionamiento de NN.UU. Esta es una de las cuestiones que, como las que siguen, se desprende de las últimas Memorias sobre la actividad de la Organización.

\section{NUEVAS ACTMIDADES Y ENFASIS EN TEMAS DE PAZ Y EN LA REESTRUCTURACION FUNCIONAL DE LA ORGANIZACION}

1. Aumenta el volumen de nuevas actividades en curso y la variedad, especificidad y capacitación técnica requerida para cada situación. Mientras que la Organización prosigue misiones que se iniciaron en los años cuarenta, recibe al mismo tiempo un número creciente de presiones de los estados para incrementar estas actividades. El trabajo de NN.UU. en el conflicto Iraq-Kuwait sirve para ilustrar el nuevo carácter de éstas: sólo la misión de observación militar decidida por el Consejo se ajusta al carácter habitual de las misiones de mantenimiento de la paz; el resto [creación de una comisión para la demarcación de la frontera entre los dos estados, eliminación de armas de destrucción en masiva iraquíes mediante una comisión especial y el Organismo Internacional de la Energía Atómica (OIEA), administración de un fondo de indemnización, y gestión de la restitución de los bienes kuwairies apropiados por Iraq] constituyen nuevos tipos de responsabilidades, sin precedentes en el trabajo internacional de NN.UU.

2. Temas relativos al capírulo VII: el propio conflicto del Golfo mostró las consecuencias indirectas de la puesta en práctica de las decisiones del Consejo de Seguridad: la imposición de sanciones económicas a un estado tiene consecuencias directas para terceros que realicen habitualmente intercambios comerciales intensos, normalmente por motivos de proximidad, con el estado 
sancionado ${ }^{4}$. Se deben estudiar las formas de asegurar la proporcionalidad en el uso de la fuerza en la aplicación de resoluciones, y el cumplimiento de las normas de derecho humanitario aplicables en los conflictos armados en los casos en que el uso de la fuerza es ejercido no por una fuerza de NN.UU., sino por coaliciones internacionales que actúan con arreglo a resoluciones del Consejo. Los organismos y acuerdos regionales de seguridad deberán actuar de forma complementaria y no concurrente con los de la Organización; de otro modo, la fragmentación que implica la regionalización irá en detrimento de la efectividad de ambos sistemas.

3. Los dos últimos Secretarios Generales han dedicado gran atención a la cuestión de la prevención y resolución de conflictos, respecto a la cual señatan una serie de necesidades:

- El desarrollo de un sistema de diplomacia preventiva, lo que a su vez presupone capacidad de alerta rápida (early warning) e información $\mathrm{fI}_{\mathrm{I}}$ dedigna y de fuentes independientes. En la agenda del Consejo deben figurar no únicamente los temas de paz inscritos a solicitud del estado o estados interesados, sino que el Secretario General y el Secretariado deben ser activos en este campo.

- El fortalecimiento de la capacidad de mediación de la Organización, que, anres de que se hayan producido actos de agresión, puede "contribuir a reconciliar reclamaciones o intereses legítimos y conseguir soluciones justas y honorables» (Pérez de Cuellar, 1991:10); así como un mayor recurso al Tribunal Internacional de Justicia (TIJ), al que deberían acudir tanto los estados (posibilidad muy poco explotada), como la Asamblea y el Consejo (art. 96 de la Carta).

- El avance en tareas no sólo de mantenimiento de la paz (peace-keeping), sino de imposición/restablecimiento de la misma (peace-making) para casos en los que no funcionen las medidas preventivas; ello significa aumento del carácter coercitivo de las misiones.

- Dotar al trabajo de las misiones de mayor seguridad y previsibilidad (seguridad financiera, a través del compromiso de los estados en el pago de contribuciones, y previsibilidad de los efectivos). En general, los estados están fácilmente dispuestos a aportar tropas, pero el pro-

4. Es por ello que al are. 80, que regula el tema, debería ser completado con acuerdos por los cuales se obligue a ofrecer asistencia al o a los terceros así afectados.

5. Pérez de Cuéllar quiere así profundizar en la relación de complementariedad entre Asamblea, Consejo y TIJ, y reafirmar al mismo tiempo la importancia de la función intermediadora del Secretario General y la primacia del derecho internacional como base de las actividades de la Organización y como rector de las relaciones internacionales. 
blema es conseguir el apoyo logístico necesario: policía, personal civiI para la organización de elecciones, etc. Afirma Ghali (1992b:28) que sería deseable que los estados indicasen claramente con qué están dispuestos a contribuir (como figura en los arts. 43 y 47.2 de la Carta).

4. Ghali emprende la tarea de simplificación, reestructuración y racionalización de la Secretaría. Objetivos: evitar duplicidades, definición de las líneas de decisión y mejora de la integración de las actividades en la esfera económica y social.

La estabilidad financiera es una demanda reiterada por los altos funcionarios de la Organización. Se dan diversas situaciones especialmente paradójicas: la aprobación del presupuesto por consenso (resolución 41/213 de la Asamblea) debería comportar también el compromiso de los estados en el pago de sus cuotas, cosa que no siempre ocurre. Mientras crecen las peticiones de actividad de NN.UU., sus ingresos permanecen bajo mínimos. Se arrastran déficits perennes debidos a la falta de cumplimiento de estas obligaciones: en septiembre de 1992, sólo cincuenta y dos estados estaban al corriente de los pagos, y quedaban pendientes 908,5 millones de dólares de cuotas más 844,4 de operaciones de mantenimiento de la paz. Ghali ha propuesto diversas medidas (1992a:18-22) para salir de esta situación: entre otras, el cobro de intereses sobre los atrasos, la flexibilización de los mecanismos de préstamo y retención de excedentes, y la creación de diversos fondos y reservas que permitan disponer de capital de forma inmediata.

5. ¿Se olvidan los temas de desarrollo? Por una parte, los conceptos manejados son los correctos: se habla de que la pobreza udebilita paulatinamente la cohesión de las sociedades y los estados, destruye la base de los derechos humanos y perjudica la salubridad del medio ambienten (Pérez de Cuéllar, 1991:16). Se pone de manifiesto la utilidad de indicadores como el del Desarrollo Humano, que dé idea no sólo de la renta per cápita, sino también del reparto de ésta y de las condiciones de vida (acceso a la educación, seguridad, participación en las decisiones) de la población; la necesidad del trabajo a largo plazo; la imposibilidad de los derechos humanos sin desarrollo; la relación directa del tema con la seguridad; la necesidad del cambio del modelo de cooperación intergubernamental, de que las ayudas sean realmente al desarrollo, y no se condicionen a la compra de determinados bienes producidos por el donante; y la deseabilidad de una mayor coordinación entre el ECOSOC y las instituciones de Bretton Woods. Se afirma la necesidad de un acercamiento integrado y pluridimensional, que supere la fragmentación sectorial, al desarrollo; y ello desde los propios organismos especializados. Los informes anuales, por ejemplo, del Banco Mundial, del Fondo de las NN.UU. para la Po- 
"Papers": Revista de Sociologia

blación, y del Programa de las NN.UU. para el Desarrollo (PNUD) se refieren a la inevitable imbricación de los temas relevantes para cada uno de ellos.

Por otra parte, la realidad es que el trabajo de NN.UU. en estas cuestiones no ha sufrido ninguna transformación, ni su volumen ha crecido sustancialmente. Quedan, pues, como temas pedientes, que esperan, como afirmaba Bertrand, el impulso de actores con capacidad de liderazgo, y el acuerdo posterior sobre su alcance y funcionamiento.

\section{OTRAS CARPETAS ABIERTAS}

Aparte de las observaciones de los altos funcionarios, NN.UU. tiene planteados, a corto y medio plazo, una serie de temas y necesidades no sosiayables:

1. El aumento del carácter político del Secretario General, por encima de uno meramente funcionarial (aunque de hecho sea éste el que le atribuye la Carta) sería básico para aumentar su efectividad de mediación y acción en temas de seguridad. Si él debe llevar adelante el papel de coordinación e integración del trabajo de NN.UU., precisa igualmente dominar el Comité Administrativo de Coordinación (CAC), órgano que reúne a los directores ejecutivos de las diversas agencias del sistema ${ }^{6}$ -en él, pese a ejercer la presidencia, el Secretario General no es más que un primus inter pares. 1

2. Existen numerosas esferas en las que NN.UU. debe trabajar a largo plazo. Dotar a la Organización de los recursos económicos que le corresponden sería el primer paso imprescindible con el qute los estados pueden manifestar su interés en tareas no sólo reactivas. Es claro que una política de desarrollo seria, encaminada a crear estructuras perdurables, debe ser planteada de este modo.

3. La actividad de arreglo pacífico de controversias ha conocido importantes logros. No obstante, la seguridad depende hoy de factores no estrictamente militares, y si NN.UU. quiere avanzar en el carácter preventivo del mantenimiento de ésta, se debe realizar un importante desarrollo conceptual, que identifique, trate e integre dichos factores susceptibles de convertirse en amenazas. En un momento en que el poder económico desaffa al militar, se plantea asimismo la actualización de la lista de potencias principales, incorporando al Consejo de Seguridad aquellos es-

6. A esta conclusión se llega también el texto de Brian Urquhart y Erskine Childers, que constituye una revisión a fondo del papel del Secretario General y de los criterios de selección de éste y del funcionariado de la Organización. Su argumento principal: la situación de necesidad de personas con capacidad de dirección y liderazgo en que se encuentra NN.UU. 
tados cuyo poder económico les otorga, de hecho, una responsabilidad equiparable al resto de miembros permanentes del órgano. La entrada en el Consejo de Japón y la RFA dependerá de diversos factores: entre otros, la voluntad de Tokyo de empezar a jugar un papel político mundial relevante, y de la CE de actuar con una sola voz en política internacional. 4. Si en 1945 se ideó la fórmula de la "responsabilidad especial" para referirse al papel de los miembros permanentes del Consejo, hoy es un hecho que $i$. la pobreza constituye un gravísimo factor de amenaza para la paz y la seguridad, ii. por diversos factores (la revolución tecnológica en primer lugar), los países pobres son cada vez más prescindibles en términos de economía global. La capacidad de producción y de consumo de sus poblaciones hace que su relevancia sea decreciente, y que el sistema de la economía mundial tienda a dejarlos fuera; y iii. esta tendencia se retroalimenta, ayudada por las barreras a la exportación, las subvenciones a la agricultura, y el aumento del comercio y de los organismos de intercambio Norre-Norte. Es por ello que esta responsabilidad especial se extiende a la práctica totalidad de estados desarrollados. En esta línea, el PNUD toma la idea de Bertrand y propone un "Consejo de Seguridad del Desarrollo".

5. El Sur ha sido sorprendido por el nuevo papel de NN.UU. y por la reestructuracion de su Secretaría, que ha contemplado con ciertos recelos $^{7}$. Si su respuesta es la vuelta a los viejos argumentos (igualdad soberana de los estados para negociar y no injerencia en los asuntos internos para temas de minorías y derechos humanos), los temas de su agenda no prosperarán. Por otra parte, su cohesión como grupo se ha visto disminuida con el debilitamiento del Movimiento de los no alineados y con el crecimiento de las ya enormes diferencias económicas entre sus miembros.

6. NN.UU. se enfrenta a nuevos cometidos para los que no fue inicialmente concebida. Cuenta para ello con atriburos considerables: es un foro intergubernamental que ha generado niveles importantes de internacionalidad, entendida ésta como la capacidad de trabajo - sobre todo de sus funcionarios- en defensa del interés de la Organización. Cuenta también con un importante corpus jurídico: instrumentos aplicables a derechos humanos, desarme, o prevención de la discriminación, que permiten que sus actividades hallen apoyo en el Derecho Internacional.

7. Dos versiones diferentes de una misma organización: South Centre (cuya misión es el fortalecimiento de la cooperacion y solidaridad Sur-Sur): en Enhancing the Economic Role of the United Nations, se anima a los países del Sur a estudiar estos cambios y apoyatios en su caso; en The United Nations at a Critical Crossroad - Time for the South to Ace se critica la decision unilateral de la que son fruto y se anima al Sur a hacer sus propias propuestas. 
De estas nuevas tareas, una es la más destacable: la de facilitar la transición, política y económica. Corresponde a Naciones Unidas el impulso de una sociedad mundial que promueva la integración de los países en desarrollo y en transición política.

\section{CONCLUSION}

Pese al hecho de que las consecuencias del fin de la guerra fría son ya notorias en múltiples esferas, la reversibilidad de la apertura del régimen de Rusia, el fin de la reforma interna y la vuelta a formas autoritarias no son descartables; quedan igualmente por cerrar temas como los acuerdos de desarme bilateral EUA-Rusia, o de regulación de las armas de destrucción masiva; otros relativos al status nuclear de determinadas repúblicas de la antigua URSS; o medidas para evitar la proliferación nuclear tras la desaparición de ese estado. Todo ello hace que Naciones Unidas tenga aún en su agenda problemas de seguridad relativos al orden bipolar, al tiempo que ya se han añadido otros causados por la desaparición del liderazgo soviético y de la ideología comunista como factor de cohesión. La urgencia y gravedad inmediata de problemas como la Guerra del Golfo y sus consecuencias, el conflicto yugoslavo o las tensiones en las nuevas repúblicas independientes tras la disgregación de la URSS han hecho que, en cierto modo, hayan pasado a un segundo plano las cuestiones relativas al desarrollo y la interdependencia económica que tanta fuerza parecían tener a finales de la pasada década. La labor de Naciones Unidas no podrá alcanzar mayor estabilidad y previsibilidad en tanto no se cierre parte de las carpetas abiertas.

El tema que sí parece fijado después del fin de la guerra fría es el de la extensión del modelo político, cultural y económico occidental al conjunto de la sociedad internacional. La toralidad de ésta se ha constituido en estados independientes, de estructuras dispares aunque cada vez más homologables; estados con cohesiones internas variables, especialmente bajas en determinados territorios a los que se impusieron formas de descolonización desde la metrópoli. Mientras que los estados del Norre se aproximan (pareciéndose cada vez más en términos políticos y económicos), los del Sur se fragmentan, tanto que su simple consideración como grupo parece impropia. La diferencia, tanto en términos de renta como de capacidad de exportación, entre los países del África subsahariana y los del Sudeste asiático es disparatada.

El Sur ve cómo desde NN.UU. se le reclama respeto a los derechos humanos y a los de sus minorias; desde las instituciones de Bretton Woods, se le imponen políticas de ajuste; desde las organizaciones regionales económicas del Norte se limita su capacidad exportadora. La posguerra fría viene de la mano 
de la intensificación en los intercambios Norte-Norte, la fragmentación en subsistemas económicos regionales, liderados por diferentes potencias económicas y la reorientación de la ayuda oficial al desarrollo de los países industrializados, sobre todo en función de sus intereses en el subsistema al que pertenecen.

El empobrecimiento progresivo de una parte creciente de las poblaciones de los países más pobres es un hecho, como lo es el carácter anti-sistema, antioccidental, que toma la revuelta social en esos países, en los que la crisis económica se transforma en cuestionamiento de la política y de las formas occidentales. La crisis del desarrollo, que está en relación directa con los niveles de formación y capacitación, muestra la crisis de la política como medio de transformación de la sociedad.

Tras un largo liderazgo bicéfalo, el mundo parece entrar en una fase en la que se combina globalización en los problemas y fragmentación en las soluciones adoptadas. Naciones Unidas puede incorporar ese elemento de liderazgo y transformarse en un foro global, no sólo para el tratamiento de problemas mundiales, sino también para que el Norte halle formas para compensar, a través de la cooperación, los costos sociales que la extensión de su hegemonía y su modelo ocasionan a poblaciones de todo el mundo. Decía Pérez de Cuéllar en su última Memoria: "Se trata de saber si, por sus decisiones y acciones, las Naciones Unidas conservan la confianza de los pueblos de todas las culturas y confinentes" (1991:28). Hasta ahora, parece que la respuesta aún es sí; pero es importante que la Organización aparezca no como la legitimadora de un cierto orden mundial, sino como un acror flexible que favorezca la transición hacia él.

\section{ANEXO 1}

Misiones de mantenimiento de la paz

\subsection{Descripción}

Se describen las 13 operaciones de Naciones Unidas de mantenimiento de la paz por nombre, fecha de establecimiento, número de efectivos y mandato general.

Operación de las Naciones Unidas en Somalia (ONUSOM), 24 de abril de 1992,

50 observadores militates y una fuerza de seguridad de 500 efectivos que se desplegarán posteriormente; para facilicar la cesación inmediata de hostilidades, mantener el alto el fuego en Mogadiscio, promover la reconciliación y el arreglo político y proporcionar asistencia de carácter humanitario.

Autoridad Provisional de las Naciones Unidas en Camboya (APRONUC), 28 de febrero de 1992, 15.000 efectivos militares y civiles; supervisat la transición hacia un nuevo gobierno después de celebrar elecciones multipartidistas. 
Fuerza de Protección de las Naciones Unidas (UNPROFOR), 21 de febrero de $1992,13.000$ efectivos militares y civiles; asegurar las condiciones de paz necesarias para negociar la solución general de la crisis yugoslava.

Misión de Verificación de las Naciones Unidas en Angola (UNAVEM II), 30 de mayo de 1991,750 policias militares y personal de elecciones; realizar nuevas tareas de verificación derivadas de los "Acuerdos de paz para Angola" hasta que terminan las elecciones generales.

Misión de Observadores de las Naciones Unidas en el Salvador (ONUSAL), 20 de mayo de 1991, 625 efectivos militares y civiles; vigilar la situación de los derechos humanos, vigilar el cumplimiento de cesación de fuego de 31 de diciembre de 1991 y vigilar el mantenimiento del orden público mientras se organiza una nueva policía nacional.

Misión de las Naciones Unidas para el Referéndum del Sáhara Occidental (MINURSO), 29 de abril de 1991, 350 efectivos militares y de apoyo; vigilar el cumplimiento del plan de arreglo, incluida la supervisión del acuerdo de cesación de fuego entre Marruecos y el Frente Polisario, y organización de un referéndum sobre el futuro del Territorio.

Misión de Observación de las Naciones Unidas para Iraq y Kuwait (UNIKOM), 9 de abril de 1991, alrededor de 460 efectivos militares y de apoyo; vigilar la frontera entre Iraq y Kuwait.

Fuerza Provisional de las Naciones Unidas en el Líbano (FPNUL), 19 de marzo de 1978, 5.854 efectivos militares; confirmar el retiro de las fuerzas israelíes del Libano meridional y ayudar al Gobierno del Líbano a restablecer la autoridad efectiva en esa zona.

Fuerza de las Naciones Unidas de Observación de la Separación (FNUOS), 3 I de mayo de 1964, 1.293 efecrivos; mantener y supervisar la cesación del fuego en la zona del Golán entre Israel y la República Arabe Siria.

Fuerza de las Naciones Unidas para el Mantenimiento de la Paz en Chipre (UNFICYP), 4 de marzo de 1964, alrededor de 2.200 efectivos militares y civiles; impedir que resurjan las hostilidades, ayudar a mantener la ley y el orden y promover el rerorno a condiciones normales.

Grupo de Observadores Militares de las Naciones Unidas en la India y el Palistán (UNMOGIP), 24 de enero de 1949, 39 observadores; supervisar la cesación del fuego entre la India y el Pakistán en la zona de Jamú y Cachemita.

Organismo de las Naciones Unidas para la Vigilancia de la Tregua (ONUVT), 11 de junio de 1948, 290 efectivos militares; supervisar diversas treguas entre árabes e israelies; actualmente presta asistencia a la FPNUL y a la FNUOS.

\subsection{Comparación de las misiones $1987-1992$}

Operaciones en funcionamiento

Personal militar destinado

Fuerzas policiales

Civiles empleados 


\section{ANEXO 2}

Los 181 estados miembros de Naciones Unidas (abril de 1993) $y$ su año de ingreso. ("Miembro originario)

Afganistán, 1949

Albania, 1955

Alemania, 1973

Angola, 1976

Antigua y Barbuda, 1981

Arabia Saudita*, 1945

Argelia, 1962

Atgentina*, 1945

Armenia, 1992

Australia*, 1945

Austria, 1955

Azerbaiyán, 1992

Bahamas, 1973

Baharein, 1971

Bangladesh, 1974

Barbados, 1966

Bélgica*, 1945

Belice, 1981

Benin, 1960

Bhután, 1971

Bielorrusia*, 1945

Bolivia* 1945

Bosnia y Hercegovina, 1992

Botswana, 1966

Brasii*, 1945

Brueni Darussalam, 1984

Bulgaria, 1955

Burkina Faso, 1960

Burundi, 1962

Cabo Verde, 1975

Camboya, 1992

Camerún, 1960

Canadá*, 1945

Colombia*, 1945

Comoras, 1975

Congo, 1960

Costa de Marfil, 1960

Costa Rica*, 1945

Croacia, 1992

Cuba*, 1945

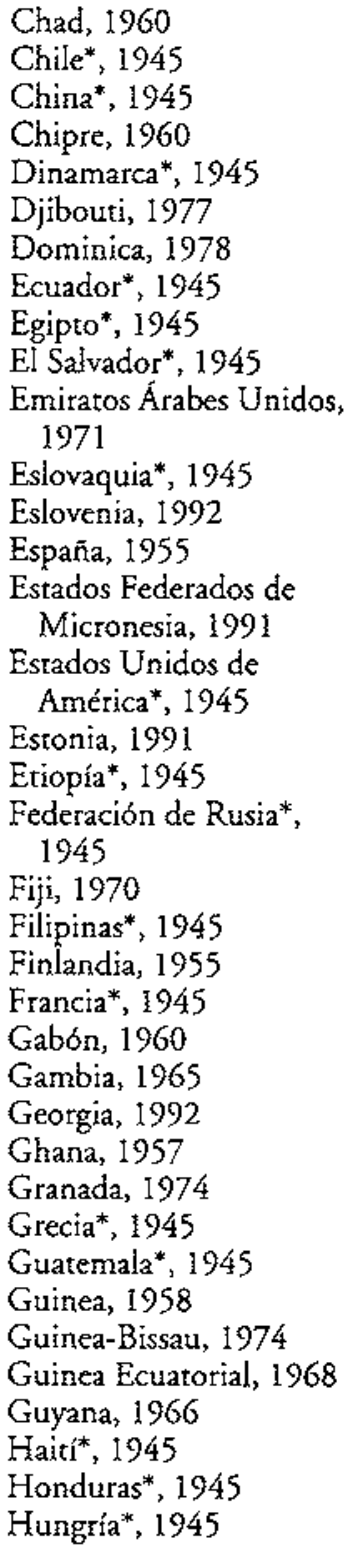

India*, 1945

Indonesia, 1950

Irán*, 1945

Iraq* ${ }^{*}, 1945$

Irlanda, 1955

Islandia, 1946

Islas Marshall

Islas Salomón, 1978

Israel, 1949

Italia*, 1945

Jamahiriya Árabe Libia, 1955

Jamaica, 1962

Japon, 1956

Jordania, 1955

Kampuchea Democrática, 1955

Kazajistán, 1992

Kenya, 1963

Kirguistán, 1992

Kuwait, 1963

Lesotho, 1966

Letonia, 1991

Líbano*, 1945

Liberia*, 1945

Liechtenstein, 1990

Lituania, 1991

Luxemburgo ${ }^{*}, 1945$

Macedonia, 1993

Madagascar, 1960

Malasia, 1957

Malawi, 1964

Maldivas, 1965

Mali, 1960

Malta, 1964

Marruecos, 1956

Mauricio, 1960

Mauritania, 1961

México*, 1945

Mongolia, 1961

Mozambiqque, 1975 
"Papers»: Revista de Sociologia

\begin{tabular}{|c|c|c|}
\hline Myanmar, 1948 & Rep. de Corea, 1991 & Sudán, 1956 \\
\hline Namibia, 1990 & Rep. de Moldova, 1992 & Suecia, 1946 \\
\hline Nepal, 1955 & Rep. Popular Lao, 1955 & Suriname, 1975 \\
\hline Nicaragua*, 1945 & Rep. Dominicana*, 1945 & Swazilandia, 1968 \\
\hline Níger, 1960 & Rep. Popular & Tayikistán, 1992 \\
\hline Nigeria, 1960 & Democrática de Corea, & Tailandia, 1946 \\
\hline Noruega*, 1945 & 1991 & Togo, 1960 \\
\hline Nueva Zelanda*, 1945 & Rumania, 1955 & Trinidad y Tobago, 1962 \\
\hline Omán, 1971 & Rwanda, 1962 & Túnez, 1956 \\
\hline Países Bajos*, 1945 & Samoa, 1976 & Turkmenistán, 1992 \\
\hline Pakistán, 1947 & San Kitts y Nieves, 1983 & Turquía*, 1945 \\
\hline Panamá*, 1945 & San Marino, 1992 & Ucraniz*, 1945 \\
\hline Papúa Nueva Guinea, & Santa Lucía, 1979 & Uganda, 1962 \\
\hline 1975 & Santo Tomé y Príncipe, & Uruguay* $^{*}, 1945$ \\
\hline Paraguay*, 1945 & 1975 & Uzbekistán, 1992 \\
\hline Perü* 1945 & San Vicente y las & Vanuatu, 1981 \\
\hline Polonia*, 1945 & Granadinas, 1980 & Venezuela*, 1945 \\
\hline Portugal, 1955 & Senegal, 1960 & Viet Nam, 1977 \\
\hline Qatat, 1971 & Seychelles, 1976 & Yemen, 1947 \\
\hline Reino Unido*, 1945 & Sierra Leona, 1961 & Yugoslavia*, 1945 \\
\hline Rep. Árabe Siria*, 1945 & Singapur, 1965 & Zaire, 1960 \\
\hline Rep. Centroafricana, & Somalia, 1960 & Zambia, 1964 \\
\hline Rep. Checa*, 1945 & $\begin{array}{l}\text { Sr1 Lanka, 195) } \\
\text { Sudáfrica* } 1945\end{array}$ & \\
\hline
\end{tabular}

\section{BIBLIOGRAFÍA.}

Banco Mundial (1992), Desarrollo y Medio Ambiente. Informe 1992. Publicaciones del Banco Mundial, Washington, 1992.

Bertrand, M. (1989a), The Third Generation World Organization. Martinus Nijhoff, Dordrecht.

Bertrand, M. (1989b), "The Process of Change in an Interdependent World and Possible Institutional Consequences" en Renninger, Y.P. Op. cit. pp. 39-73.

Butros Ghali, B. (1992a), Memòria del Secretari General sobre l'activitat de l'Organització 1992. Barcelona, Associació per a les NN.UU. a Espanya - Centre Unesco de Catalunya.

Butros Ghali, B. (1992b), Un programa de Patu. Barcelona, Associació per a les NN.UU. a Espanya - Centre Unesco de Caralunya.

Butros Ghali, B. (1992c), «L'ONU en première ligne», Politique internationale, núm. 57, otoño 1992, pp. 143-149.

Groom, A.J.R. (1988), "The Advent of international Organisation" en Taylor, P. y Groom, A.J.R. (1988), Op. cit. pp. 3-21. 
Naciones Unidas (1990), $A B C$ de las Naciones Unidas. Nueva York, Dep. de Información Pública de NN.UU.

Pérez de Cuéllar, J. (1988-1991), Memòria del Secretari General sobre l'activitat de l'Organitzaci6. Barcelona, Associació per a les NN.UU. a Espanya - Centre Unesco de Catalunya.

Programa de las NN.UU. para el Desarrollo (1992), Informe Mundial 1992. Bogotá, Tercer Mundo Editores.

Renninger, J. P. (compilador) (1989), The Future Role of the United Nations in an Interdependent World. Dordrecht, Martinus Nijhoff.

Salomón, M. (1991), Les Nacions Unides entre la crisi i la consolidació. Estudis Internacionals 1/1991. Barcelona, Centre Unesco de Catalunya - Centre d'Estudis sobre la Pau i el Desarmament (UAB).

South Centre (1992), Enhancing the Economic Role of the United Nations. Ginebra, South Centre.

South Centre (1992), The United Nations at a Critical Crossroads. Ginebra, South Centre.

Stockholm Initiative on Global Security and Governance (1991), Common Responsibility in the 1990's. Estocolmo, Oficina del Primer Ministro.

Taylor, P. y Groom, A.J.R. (compiladores) (1988), International Institutions at Work. Londres, Pinter.

Taylor, P. y Groom, A.J.R. (compiladores) (1989), Global Issues in the United Nations' Framework. Londres, McMillan.

Urquharc, B. y Childers, E. (1990), Un mundo en necesidad de conduccion: las Naciones Unidas del mañana. Uppsala, Fundación Dag Hammarskjol. 